\section{Avaliação da implementação da assistência ao planejamento reprodutivo em três municípios do Estado do Rio de Janeiro entre 2005 e 2007}

\section{An evaluation of the implementation of family planning assistance in three municipalities in the State of Rio de Janeiro, Brazil, between 2005 and 2007}

Claudia Bonan 1

Kátia Silveira da Silva 2

Ana Lúcia Tiziano de Sequeira 3

Márcia Cristina Rodrigues Fausto 4

1-3 Instituto Fernandes Figueira. Fundação Oswaldo Cruz. Av. Rui Barbosa, 716. Flamengo. Rio de Janeiro, RJ, Brasil. CEP 22.250020. E-mail: cbonan@globo.com

4 Escola Nacional de Saúde Pública. Fundação Oswaldo Cruz. Rio de Janeiro, RJ, Brasil.

\begin{abstract}
Objectives: to evaluate the implementation of family planning (FP) assistance in three municipalities in the State of Rio de Janeiro, Brazil, along with contextual factors.

Methods: the evaluation was carried out between 2005 and 2007. Interviews were conducted with health workers and managers, the provision of services was observed, and documents consulted. The degree of implementation of the program was assessed by developing an evaluation matrix comprising three components - assistance, education and management - and standards and criteria were established. Oral and documentary sources of information allowed for analysis of the context to the implementation to be carried out.

Results: the implementation of FP in the metropolitan municipality was at an advanced stage and in the rural municipalities in its initial stages. Educational activities conformed better to the established criteria than clinical care. Weaknesses included irregular methods of contraception, problems with the availability of contraceptive surgery, the lack of infertility care and male reproductive health. The teams include a range of different professionals, but there is a need for further training. In the metropolitan municipality, program management is a strong point, but in the others management falls short of expectations.

Conclusions: the degree of implementation of FP care is related to geopolitical inequalities and contextual factors associated with the Brazilian National Health System-lack of integration of care networks and limited regionalization. This study may help to encourage institutionalization of the practice of monitoring and evaluating family planning assistance.
\end{abstract}

Key words Familiar planning (Public Health) Reproductive rights, Programs evaluation

\section{Resumo}

Objetivos: avaliar a implementação das ações de assistência ao planejamento reprodutivo (PR) em três municipios fluminenses e os aspectos contextuais.

Métodos: pesquisa avaliativa realizada entre 2005-2007. Foram realizadas entrevistas com gestores e profissionais de saúde, observação de serviços e análise de documentos. Para análise do grau de implementação do programa foi desenvolvida matriz de avaliação com três componentes assistência, educação e gerenciamento - e estabelecidos padrões e critérios. Fontes orais e documentais possibilitaram análise do contexto de implementação.

Resultados: a implementação do PR no município metropolitano está avançada e nos municípios do interior é incipiente. Atividades educativas apresentaram maior conformidade com critérios do que atividades clínico-assistenciais. Irregularidade de métodos contraceptivos, problemas na oferta de contracepção cirúrgica, ausência de atenção à infertilidade e à saúde reprodutiva masculina foram aspectos frágeis. Existem equipes multiprofissionais, mas há necessidade de capacitação. No municipio metropolitano, o gerenciamento do programa é ponto forte, mas nos demais é débil.

Conclusões: o grau de implementação da assistência ao PR se relacionou com desigualdades geopolíticas entre os municípios e aspectos do contexto institucional do SUS - pouca integração da rede assistencial e regionalização limitada. O estudo pode contribuir para institucionalização da prática de monitoramento e avaliação das ações de assistência ao planejamento reprodutivo.

Palavras-chave Planejamento familiar, Direitos reprodutivos, Avaliação de programas 


\section{Introdução}

Nas últimas três décadas, o Estado brasileiro assumiu crescente compromisso com a promoção dos direitos reprodutivos de mulheres e homens, como resultado de um conjunto de processos políticos que marcaram a conjuntura nacional e internacional. Instrumentos jurídicos e normativos criados nesse período - o Programa de Assistência Integral à Saúde da Mulher (1984), a Constituição Federal (1988), a Lei de Planejamento Familiar (1996), a Política Nacional de Assistência Integral à Saúde da Mulher (2004) e a Política Nacional de Direitos Sexuais e Direitos Reprodutivos (2005) estabeleceram diretrizes para uma política de saúde sexual e reprodutiva, baseada nos princípios do direito, da equidade e da integralidade. Ao longo da construção do Sistema Único de Saúde (SUS) e do processo de descentralização, tem se estruturado a área da assistência à saúde sexual e reprodutiva nos estados e municípios brasileiros, sendo esta já razoavelmente estabelecida.1-3

Em que pese os avanços observados, persistem problemas no que se refere ao acesso e à qualidade da assistência prestada à saúde sexual e reprodutiva, incluindo o planejamento reprodutivo, objeto do estudo apresentado neste artigo.2-5 A pesquisa nacional de demografia e saúde da criança e da mulher 2006 evidenciou melhoras no acesso das mulheres brasileiras à contracepção e a serviços de saúde sexual e reprodutiva, mas também mostrou a permanência de disparidades significativas entre grupos populacionais, segundo escolaridade, cor da pele, área de residência urbana ou rural e região do país, indicando a persistência de desigualdades sociais em saúde. ${ }^{1}$ Estudos recentes demonstram que as dificuldades de implementação efetiva da política de assistência ao planejamento reprodutivo se relacionam tanto com tarefas que competem ao gestor federal, como com aquelas da gestão estadual e municipal.2-10

Neste artigo apresentamos os resultados de uma pesquisa avaliativa sobre a implementação da assistência ao planejamento reprodutivo em três municípios do Estado do Rio de Janeiro, cujos objetivos foram avaliar o grau de implementação dessas ações programáticas e investigar como aspectos do contexto político, cultural e institucional poderiam influenciar nesse processo. Apoiamo-nos em um arcabouço teórico que compreende a construção de políticas de saúde como um processo complexo, que envolve disputas políticas e interações entre um espectro amplo de atores - estatais e não-estatais, nacionais, locais e globais, administradores, técnicos e demandantes - e momentos formativos analiticamente distintos: formalização de marcos normativos, desenho da intervenção (objetivos, diretrizes, prioridades, estratégias, ações e metas), planejamento e alocação de recursos e, enfim, implementação. ${ }^{11}$ Essa última é entendida como a operacionalização de uma intervenção planejada, envolvendo instalação de uma infra-estrutura e produção de serviços e bens, em um determinado contexto organizacional, político e social. ${ }^{12}$ Com base nessas proposições e na revisão da literatura sobre o assunto, partimos da premissa que a implementação é uma tarefa ainda não concluída do processo instituinte da política de assistência ao planejamento reprodutivo no Brasil.

A pesquisa é parte do projeto "Desenvolvimento das perspectivas da integralidade, da humanização e do direito no contexto da atenção ao planejamento familiar", financiado pelo Programa de Inovação e Desenvolvimento Tecnológico em Saúde Pública da Fundação Oswaldo Cruz.

\section{Métodos}

Trata-se de uma pesquisa avaliativa 13 sobre a implementação de ações programáticas de assistência ao planejamento reprodutivo (PR) em três municípios do Estado do Rio de Janeiro, selecionados com auxílio da Secretaria de Estado de Saúde do Rio de Janeiro, com base nos seguintes critérios: existência do programa de assistência ao planejamento reprodutivo, independente do tempo do mesmo; potencial de institucionalização do programa; e motivação dos gestores municipais para avançar na implementação.

Os municípios - referidos neste artigo como A, B e $\mathrm{C}$ - apresentam particularidades no que diz respeito às características demográficas e modelo de atenção básica. Situado na região metropolitana, o município A possui mais de um milhão de habitantes. Na época do estudo, contava com cerca de 120 unidades básicas de saúde de modelo tradicional (centros de saúde e postos de saúde) e a estratégia de saúde da família (ESF) cobria menos de $10 \%$ da população. Praticamente toda a rede de centro municipal de saúde (CMS) e postos de saúde (PS) oferecia assistência ao PR, no ano de 2006, mas a implantação dessas ações na ESF era incipiente.

Os municípios B e C situam-se no interior do Estado e possuem menos de 60 mil habitantes. O município $\mathrm{B}$ tem sua rede de atenção básica estruturada segundo o modelo de saúde da família, cobrindo quase $100 \%$ de sua população, e todas as unidades oferecem assistência ao PR. O município C possui uma rede mista de serviços de atenção básica, com um centro municipal de saúde, alguns postos de 
saúde e unidades de saúde da família. Somente o CMS prestava assistência ao PR, no momento da pesquisa.

Em cada município, as unidades de saúde foram selecionadas de modo a captar a diversidade da experiência de implementação do PR, optando-se por serviços localizados em áreas distintas do território municipal e, no caso do município A, serviços de porte diferenciados (policlínicas, centros de saúde e postos de saúde). Foram incluídas seis unidades no município A, quatro no B e apenas um centro municipal de saúde no $\mathrm{C}$, pois era a única unidade onde já se havia implantado a assistência ao PR.

Os dados foram coletados através de fontes orais, documentais e observacionais. Foram realizadas entrevistas com 43 profissionais de saúde (29 no município A, 9 no $\mathrm{B}$ e 5 no $\mathrm{C}$ ), incluindo os secretários de saúde dos três municípios, técnicos das secretarias de saúde, gestores e profissionais das unidades de saúde. Em cada unidade, procurou-se entrevistar todos os profissionais envolvidos na assistência ao PR, mas nem sempre foi possível por motivo de férias, licença ou por estarem ocupados devido a atendimento. Foram entrevistados no mínimo dois profissionais em cada serviço nos municípios $\mathrm{A}$ e $\mathrm{C}$ e pelo menos um no município $\mathrm{B}$, distribuídos entre as seguintes categorias: seis médicos, oito enfermeiros, oito assistentes sociais e um psicólogo. As entrevistas foram guiadas por roteiros semi-estruturados, gravadas e posteriormente transcritas. Procedeu-se também a observação das unidades de saúde para avaliar a infra-estrutura da assistência ao PR e a revisão de materiais documentais referentes ao programa (registros, fichas de inscrição, estatísticas e outros). A coleta de dados abrangeu o período entre novembro de 2005 e junho de 2007.

O modelo teórico-lógico para avaliação da implementação do programa de assistência ao PR no município (Tabela 1) foi baseado nas diretrizes ministeriais e nos princípios éticos e conceituais do direito à saúde, da integralidade e dos direitos reprodutivos. 14-16 Ele orientou a elaboração dos roteiros para coleta de dados que buscaram abarcar quatro dimensões analíticas: a) questões referentes à estrutura, aos processos de assistência e ao gerenciamento das ações de planejamento reprodutivo nas unidades de saúde, b) planejamento do gestor municipal, c) dinâmicas políticas e institucionais do município e d) elementos da cultura institucional e profissional que poderiam influenciar na implementação dessas ações.

Para a descrição das questões referentes à estru- tura, aos processos assistenciais e ao gerenciamento do programa nas unidades e no município, foram utilizadas informações obtidas por meio de entrevistas, observação e análise documental. A análise do conteúdo narrativo dos relatos de profissionais e gestores entrevistados 17 possibilitou a reconstrução do histórico do planejamento familiar no município e de sua contextualização, assim como a compreensão de elementos culturais e ideológicos que permeiam a visão dos profissionais e gestores sobre o planejamento reprodutivo.

Para uma análise quantitativa do grau de implementação do programa de assistência ao PR nos municípios, foi desenvolvida uma matriz de avaliação com padrões e critérios, tendo como componentes centrais: atividades clínico-assistenciais do $\mathrm{PR}$, atividades de informação e educação e gerenciamento do programa na unidade de saúde (Tabela 2). Atribuiu-se um valor total de 1000 pontos à matriz, distribuídos entre os vários componentes e aspectos, segundo critérios derivados do modelo teóricológico do programa. Segundo o percentual de pontos obtidos pelo município, o grau de implementação do programa de PR foi classificado como: 0-39\%, não implementado; 40-60\%, incipiente; 61-70\%, moderado; $71-80 \%$, moderadamente avançado; 81 $90 \%$, avançado; $91-100 \%$, implementado.

O estudo observou os aspectos éticos para pesquisa com seres humanos determinados pela Resolução 196/1996 do Conselho Nacional de Saúde e foi aprovado pelo Comitê de Ética em Pesquisa do Instituto Fernandes Figueira/Fiocruz.

\section{Resultados}

Grau de implementação das ações de assistência ao planejamento reprodutivo na rede de atenção básica

Os resultados da aplicação da matriz de avaliação do grau de implementação da assistência ao PR na rede de atenção básica dos municípios estão sistematizados na Tabela 3 e na Figura 1. A Tabela 3 apresenta o percentual de pontos obtidos por componente e o total por município, assim como o grau de implementação do PR, segundo os níveis de corte estabelecidos. A Figura 1 mostra esquematicamente o percentual de pontos obtidos por cada município, discriminando em cada componente as dimensões de estrutura e de processo.

O município A destacou-se por apresentar um grau de implementação moderadamente avançado, enquanto os outros dois municípios estavam na faixa considerada incipiente, sinalizando a existência de 


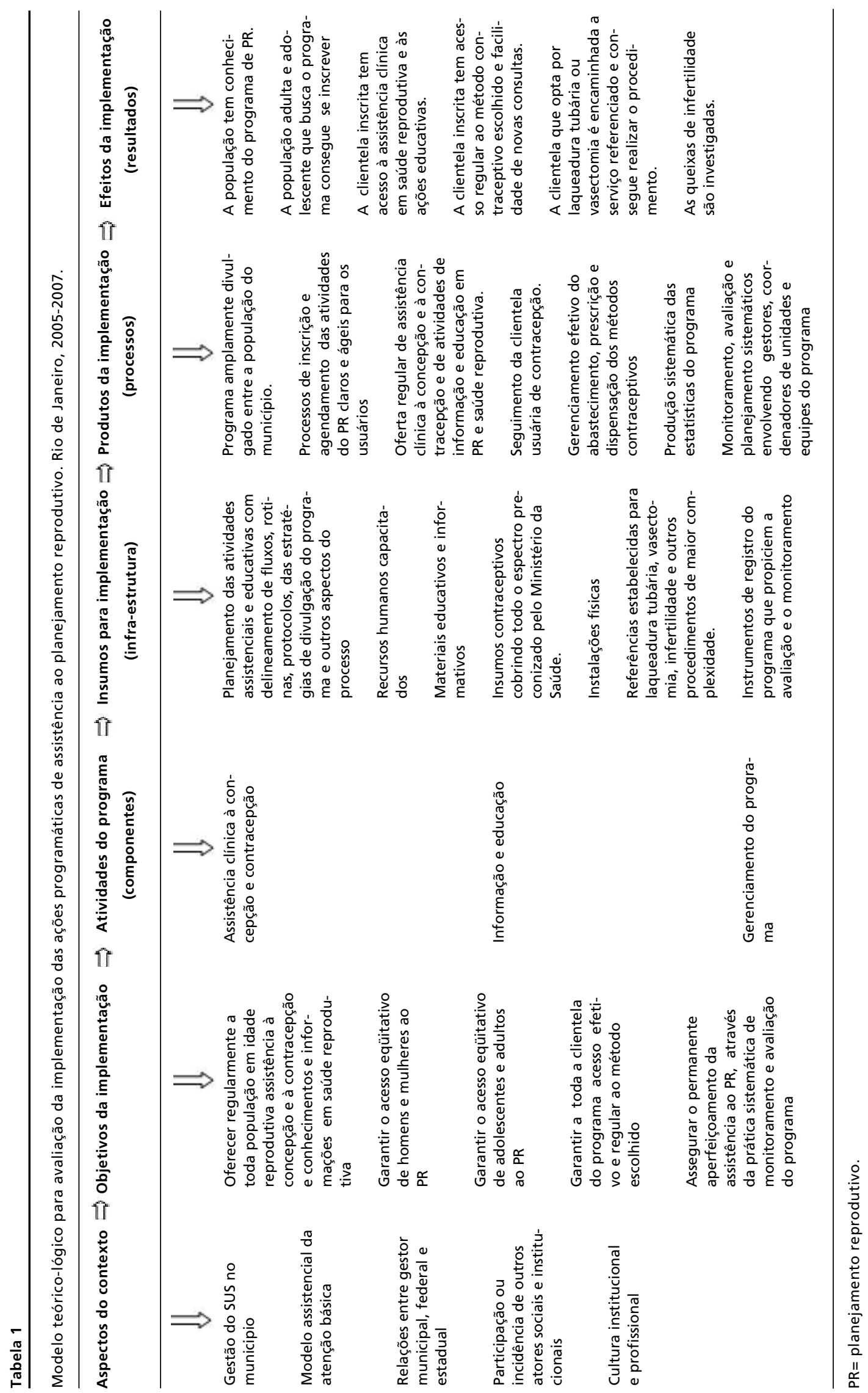

S110 Rev. Bras. Saúde Matern. Infant., Recife, 10 (Supl. 1): S107-S118 nov., 2010 
Matriz de avaliação do grau de implementação das ações programáticas de planejamento reprodutivo na rede municipal de atenção básica, segundo componentes e dimensões de estrutura e processo. Rio de Janeiro, 2005-2007.

\begin{tabular}{|c|c|c|c|}
\hline & $\begin{array}{l}\text { Atividades clínico-assistenciais do } \\
\text { planejamento reprodutivo }\end{array}$ & Informação e educação & $\begin{array}{c}\text { Gerenciamento do programa } \\
\text { na unidade }\end{array}$ \\
\hline $\begin{array}{c}\text { Aspectos } \\
\text { da } \\
\text { estrutura }\end{array}$ & $\begin{array}{l}\text { 1. Atividades planejadas para a clientela } \\
\text { (50) } \\
\text { - consulta clínico ginecológica } \\
\text { - consulta saúde reprodutiva mas- } \\
\text { culina } \\
\text { - contracepção de emergência } \\
\text { 2. Recursos humanos (50) } \\
\text { - equipe multiprofissional } \\
\text { - capacitação específica } \\
\text { 3. Métodos contraceptivos planejados } \\
\text { (50) } \\
\text { - anticoncepcional oral } \\
\text { - anticoncepcional injetável } \\
\text { - diafragma, } \\
\text { - DIU } \\
\text { - preservativo masculino } \\
\text { - preservativo feminino } \\
\text { - geléia espermicida } \\
\text { - contracepção de emergência } \\
\text { 4. Referências formalizadas (50) } \\
\text { - laqueadura tubária } \\
\text { - vasectomia } \\
\text { - investigação de infertilidade }\end{array}$ & $\begin{array}{l}\text { 1. Atividades planejadas (50) } \\
\text { - grupo educativo } \\
\text { - orientação } \\
\text { individual/casal } \\
\text { 2. Recursos humanos (50) } \\
\text { - equipe multiprofissional } \\
\text { - capacitação específica } \\
\text { 3. Instalações físicas apropri- } \\
\text { adas (20) } \\
\text { 4. Material pedagógico para } \\
\text { a atividade educativa (30) }\end{array}$ & \\
\hline $\begin{array}{l}\text { Aspectos } \\
\text { do } \\
\text { processo }\end{array}$ & 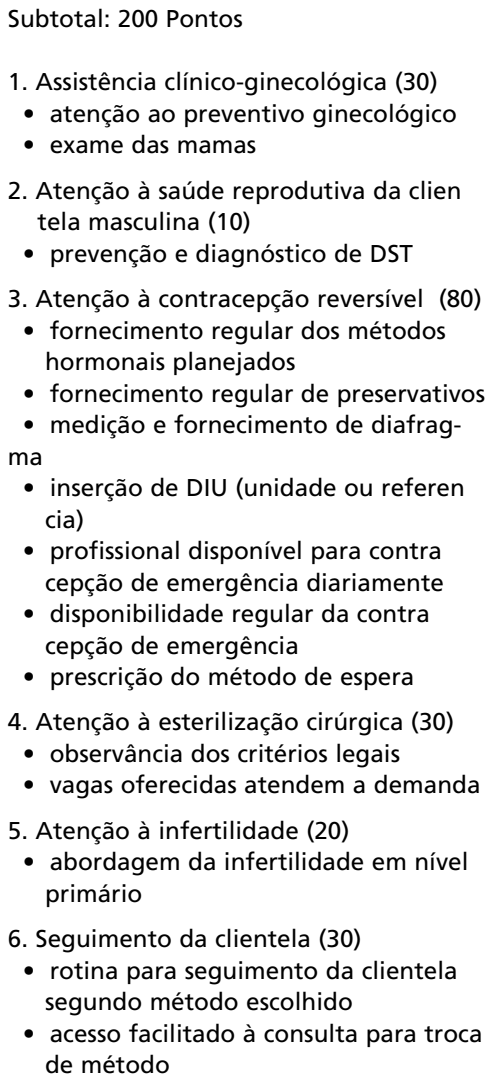 & $\begin{array}{l}\text { Subtotal: } 150 \text { Pontos } \\
\text { 1. Grupo educativo (100) } \\
\text { - periodicidade progra- } \\
\text { mada } \\
\text { - conteúdos específicos } \\
\text { - metodologia participati- } \\
\text { va } \\
\text { - oferta para toda a } \\
\text { clientela } \\
\text { 2. Orientação indivi- } \\
\text { dual/casal (50) } \\
\text { - coleta de informações } \\
\text { socioeconômicas, histó- } \\
\text { ria reprodutiva e co- } \\
\text { nhecimento e uso de } \\
\text { métodos } \\
\text { - fornecimento de infor } \\
\text { mações sobre saúde sexu- } \\
\text { al e reprodutiva } \\
\text { - registro das informações } \\
\text { em fichas próprias ou } \\
\text { prontuário }\end{array}$ & $\begin{array}{l}\text { 1. Estratégias de divulgação do } \\
\text { programa (40) } \\
\text { 2. Processo de inscrição (60) } \\
\text { - mecanismos de inscrição bem } \\
\text { estabelecidos (registro, fluxo, } \\
\text { calendário) } \\
\text { - oferta da assistência para } \\
\text { homens, mulheres, adultos e } \\
\text { adolescentes } \\
\text { - garantia de inscrição para } \\
\text { todos o que demandam } \\
\text { 3. Agendamento das atividades } \\
\text { - mecanismos que facilitem o } \\
\text { fluxo de agendamento das } \\
\text { atividades planejadas } \\
\text { 4. Produção de estatísticas (80) } \\
\text { - inscrições no programa e par } \\
\text { ticipação em grupos educativos } \\
\text { - encaminhamentos para esteri- } \\
\text { lização cirúrgica voluntária } \\
\text { - estoque e dispensação de } \\
\text { métodos } \\
\text { 5. Monitoramento (60) } \\
\text { - adequação oferta/demanda } \\
\text { - métodos adotados } \\
\text { - atendimento da clientela re- } \\
\text { ferenciada a outros serviços } \\
\text { 6. Avaliação e planejamento ( } 40 \text { ) } \\
\text { - periodicidade programada } \\
\text { envolvimento de profissionais } \\
\text { e gestores }\end{array}$ \\
\hline & & & a \\
\hline
\end{tabular}

DST=doenças sexualmente transmissíveis; DIU=dispositivo intrauterino. 
Grau de implementação das ações de planejamento reprodutivo na rede de atenção básica nos municípios, segundo componentes. Rio de Janeiro, 2005-2007.

\begin{tabular}{|c|c|c|c|c|c|}
\hline & \multicolumn{3}{|c|}{ Componentes (percentual de conformidade) } & \multirow{2}{*}{$\begin{array}{c}\text { Percentual do } \\
\text { município }\end{array}$} & \multirow{2}{*}{$\begin{array}{c}\text { Grau de } \\
\text { implementação }\end{array}$} \\
\hline & $\begin{array}{l}\text { Atividades clínico- } \\
\text { assistenciais do PR }\end{array}$ & $\begin{array}{l}\text { Informação e } \\
\text { educação }\end{array}$ & Gerenciamento & & \\
\hline Município A & $57 \%$ & $97 \%$ & $80 \%$ & $75 \%$ & $\begin{array}{c}\text { Moderadamente } \\
\text { avançado }\end{array}$ \\
\hline Município B & $41 \%$ & $55 \%$ & $32 \%$ & $43 \%$ & Incipiente \\
\hline Município C & $32 \%$ & $93 \%$ & $33 \%$ & $51 \%$ & Incipiente \\
\hline
\end{tabular}

$\mathrm{PR}=$ planejamento reprodutivo; Classificação do grau de implementação: Não implementado= 0-39\%; Incipiente=40$60 \%$; Moderado= 61-70\%; Moderadamente avançado= 71-80\%; Avançado= 81-90\%; Implementado= 91-100\%

Figura 1

Grau de implementação das ações de planejamento reprodutivo na rede de atenção básica nos municípios, segundo componentes. Rio de Janeiro, 2005-2007.

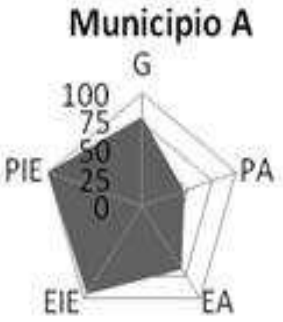

Gerenciamento do programa na unidade de saúde (G)

Processo das atividades clínico-assistenciais do PR (PA)

Estrutura das atividades clínico-assistenciais do PR (EA)

Estrutura das atividades de informaçẫo e educação (EIE)

Processo das atividades de informaçăo e educaçấo (PIE)

disparidades entre região metropolitana e interior (Tabela 3). Chama a atenção o fato do componente "Atividades clínico-assistenciais do PR" ter obtido pontuações muito inferiores àquelas do componente "Informação e educação". Os municípios interioranos não atingiram $50 \%$ de conformidade no componente assistencial e o metropolitano apenas ultrapassou esse limiar. A avaliação do componente clínico-assistencial do PR, por dimensões de estrutura e processo, mostrou que as deficiências no processo de assistência são maiores do que os relacionados à estrutura (Figura 1). Nos três casos, foram os aspectos relacionados com a atenção à contracepção reversível e à esterilização cirúrgica que mais comprometeram a implementação, destacadamente: fornecimento irregular de métodos

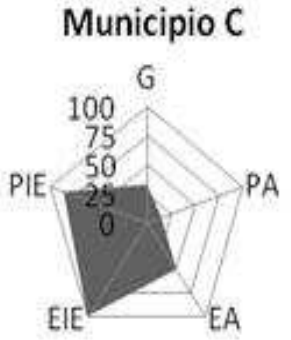

$\begin{array}{rcc}\text { Municipio A } & \text { Municipio B } & \text { Municipio C } \\ 80 \% & 32 \% & 33 \% \\ 45 \% & 32 \% & 15 \% \\ 68 \% & 51 \% & 50 \% \\ 95 \% & 54 \% & 100 \% \\ 100 \% & 57 \% & 87 \%\end{array}$

hormonais, do preservativo masculino e do diafragma; a ausência de profissional capacitado para inserir o dispositivo intrauterino (DIU), de referência formalizada para esse procedimento e/ou falta do método; fornecimento irregular do contraceptivo de emergência por falta de método ou de profissional disponível para o atendimento, no caso de A, e ausência de planejamento para esse método em B e C; ausência (município C) ou inoperância (município A) das referências para esterilização cirúrgica voluntária, baixa capacidade para atender à demanda (A e B), não cumprimento dos critérios legais para o procedimento (B). Outros aspectos que contribuíram para uma baixa pontuação do processo de assistência ao PR foram a ausência de atenção à saúde reprodutiva masculina e de abordagem das 
queixas de infertilidade, situação igual nos três municípios. O exame preventivo de câncer de colo uterino para as usuárias do PR não estava garantido em todas as unidades do município $\mathrm{A}$, nem no município $\mathrm{C}$; somente o município $\mathrm{B}$ assegurava esse procedimento a toda clientela, com sua rede básica integralmente estruturada segundo preceitos da ESF.

No que diz respeito à estrutura para as atividades clínico-assistenciais do PR, o município A teve como pontos fortes a composição de equipes multidisciplinares, um grande número de profissionais com capacitação específica e rotinas estruturadas para oferecer toda a gama de métodos contraceptivos preconizados pelas políticas ministeriais; os pontos mais débeis do programa de PR relacionaram-se com o planejamento para atender questões específicas da população masculina e pouca formalização das referências para procedimentos de maior complexidade (esterilização cirúrgica, DIU, infertilidade, exames complementares). A atenção ao PR nos municípios B e $C$ também era realizada por equipes multidisciplinares, mas muitos desses profissionais não possuíam uma capacitação específica. Nos municípios do interior, seja pela falta de profissionais capacitados para trabalhar com métodos como o DIU e o diafragma, seja pela ausência de uma política eficaz do gestor municipal, estadual ou federal no que se refere ao abastecimento de insumos contraceptivos, o planejamento das unidades de saúde se dirigia basicamente à dispensação de pílula anticoncepcional e do preservativo masculino. No município $B$, a referência para encaminhamento para esterilização cirúrgica voluntária está estabelecida, apesar dos problemas no processo conforme já mencionado.

Em relação ao componente "Informação e educação", a sua boa pontuação na avaliação com a matriz refletia um grau de sensibilização dos gestores e dos serviços para algumas das diretrizes mais importantes das políticas de saúde reprodutiva: informação e educação favorecem a autonomia e os direitos. Paradoxalmente, o município $\mathrm{B}$ que tem toda sua atenção básica estruturada no modelo da ESF foi o que teve uma menor pontuação nesse componente. Nesse município não havia um planejamento para a realização periódica de grupos educativos em PR. Conteúdos informativos referentes ao PR eram inseridos com restrição e baixa prioridade nas atividades educativas mais gerais, realizadas em grupo, sem a garantia de transmissão de conhecimentos e informações que poderiam apoiar as suas decisões e práticas

Quanto ao gerenciamento do programa de PR, os serviços do município A tiveram boa pontuação
( $80 \%$ de conformidade), com estratégias de divulgação do programa, mecanismos bem estabelecidos para a inscrição da clientela (calendário, fluxos, fichas próprias), rotinas para seguimento dos usuários, produção regular de estatísticas (inscrições, grupos educativos, métodos dispensados, encaminhamentos para esterilização cirúrgica) e prática de avaliação periódica do programa envolvendo profissionais do PR e coordenadores da unidade. Os pontos débeis foram o monitoramento da adequação oferta/demanda e dos usuários referenciados a outras unidades para realização de contracepção cirúrgica ou outros procedimentos.

Nos municípios B e C, as baixas pontuações obtidas no componente gerenciamento do programa na unidade se deveram à debilidade ou ausência de estratégias de divulgação do PR e captação da clientela, de instrumentos de registros específicos dessa assistência e, conseqüentemente, de estatísticas relativas à clientela do programa, aos métodos dispensados e aos procedimentos executados. Consequentemente, eram inexistente rotinas de monitoramento das atividades desenvolvidas pelo programa. No município B, excepcionalmente para os casos de solicitação e encaminhamento para a esterilização cirúrgica, havia registros específicos e produção de relatórios para o gestor municipal.

Na comparação do grau de implementação do PR dos municípios é necessário observar que, embora o município $\mathrm{C}$ tenha obtido uma pontuação total maior do que o $\mathrm{B}$, é importante lembrar que no $\mathrm{C}$ o programa estava implementado em uma única unidade e todos os recursos disponíveis para PR estavam concentrados nesse serviço. Por sua vez, no município $\mathrm{B}$, o processo de implementação do $\mathrm{PR}$ alcançava toda sua rede básica.

Aspectos contextuais da implementação da assistência ao planejamento reprodutivo nos municípios

A implementação das ações programáticas de PR nos municípios se dá em contextos históricos diferenciados. No município A, a implementação foi iniciada em 1992, em uma conjuntura marcada por importante mobilização em torno do Programa de Assistência Integral à Saúde da Mulher (PAISM) e do SUS, tendo à frente técnicos e profissionais de saúde com interlocução com movimentos de mulheres e de reforma sanitária. Nos anos seguintes, de modo contínuo e sustentado, fizeram-se investimentos no planejamento e na implementação das ações de assistência ao PR, tanto no que diz respeito à infra-estrutura como aos processos de trabalho, e, 
destacadamente, em capacitação de recursos humanos. A iniciativa da secretaria de saúde de criar um centro de treinamento em atenção integral à saúde da mulher, em 1992, foi crucial para a valorização do trabalho educativo, o conhecimento das leis, normas e princípios éticos do PR, a capacitação técnica dos profissionais para o gerenciamento das ações do programa nas suas unidades de saúde, além da abertura para o trabalho multiprofissional. Um permanente intercâmbio da rede de saúde com instituições de ensino e pesquisa, organismos profissionais, organizações da sociedade civil e movimentos sociais também foi um fator a fomentar esse processo.

Nos municípios B e C, o processo de implementação do PR foi iniciado mais recentemente - em 2002, em B, e em 2004 em C - e impulsionado por circunstâncias distintas do município A. Em ambos os casos, o interesse dos gestores locais, compartilhado com gestores estaduais, em implantar e/ou regularizar a assistência à esterilização cirúrgica voluntária no SUS, em conformidade com a Lei de Planejamento Familiar, foi uma grande motivação. Os municípios apresentaram projetos à Secretaria Estadual de Saúde (SES-RJ) para credenciamento dos serviços de esterilização cirúrgica, nos quais se previam também a estruturação da assistência à contracepção reversível e atividades educativas em PR.

A iniciativa de implementação do programa de assistência ao PR nesses dois municípios, na primeira metade da década de 2000 , refletia uma maior presença dos gestores estaduais que, de certa forma, foram indutores desse processo. A receptividade dos gestores municipais e dos profissionais de saúde à política ministerial de saúde sexual e reprodutiva - inclusive, preocupação de adequar-se às suas normas e diretrizes - foi também um aspecto favorável. Contudo, o suporte do gestor estadual de saúde para o desenvolvimento da assistência ao PR conforme previsto nas leis e normas nacionais ainda era limitado. Suprir as necessidades de abastecimento de métodos contraceptivos reversíveis, de infra-estrutura para a oferta de esterilização cirúrgica e de capacitação de recursos humanos apresentavase como grande desafio para os municípios, cujas prefeituras não contavam com recursos próprios para tal. Tampouco contavam com universidades, agências de cooperação técnica ou organizações da sociedade civil atuando em seus territórios que pudessem apoiá-los. Além disso, a experiência de convênios ou consórcios com os municípios adjacentes ainda eram muito incipientes.

Aspectos do contexto organizacional do SUS nos três municípios estudados revelaram também limitações para o avanço da implementação das ações de assistência ao PR. Apesar das particularidades, um aspecto comum aos municípios, foram as debilidades de integração da assistência básica com os serviços de maior complexidade, representadas pelas dificuldades de estabelecimento de mecanismos de referência e contra-referência eficazes para procedimentos de esterilização cirúrgica, investigação de infertilidade e realização de exames complementares.

Um outro aspecto limitador da implementação diz respeito a um traço da cultura institucional que reproduzia na assistência ao PR a lógica dos programas verticais. Ainda havia muitos obstáculos para a estruturação das ações programáticas de modo mais transversal, perpassando diversos serviços e momentos do contato dos usuários com as unidades de saúde. No município A, em certos aspectos, a assistência ao PR nas unidades de saúde estava estruturada como um serviço "a mais", específico, nem sempre articulado satisfatoriamente com a ginecologia, o pré-natal, o programa de DST/Aids e de adolescentes, ocasionando perda de oportunidades de captação de clientela e, por vezes, disputas de competências e conflitos. No município B, por seu turno, as ações de assistência ao PR estavam inseridas nas demais ações desenvolvidas pelas equipes de saúde da família, não tendo um caráter de um serviço à parte. Entretanto, essas ações careciam de maior estruturação programática: as rotinas da assistência ao PR eram pouco formalizadas e os fluxos pouco definidos, não havia registros específicos dos usuários do PR nas unidades de saúde (sexo, idade, métodos adotados, etc), não se produziam estatísticas do programa (clientela atendida, insumos contraceptivos dispensados e encaminhamentos feitos) e não havia mecanismos de monitoramento e avaliação estabelecidos. Manifestandose como um serviço diferenciado (município A) ou como um conjunto de ações fragmentadas (município B), o desafio de superar a tradição dos programas verticalizados e de criar uma cultura institucional de cuidados coordenados, integrados e integrais apresentava-se como um aspecto contextual que condiciona as oportunidades de implementação das ações programáticas de PR nos municípios estudados.

Outros aspectos da cultura institucional influenciaram o processo de implementação do PR. A linguagem, a mensagem e a organização dos processos de trabalho implícita ou explicitamente privilegiavam o público feminino e adulto, faltando estratégias mais elaboradas para atrair homens e 
adolescentes. O PR ou "planejamento familiar", segundo a linguagem mais corrente, era visto como contracepção; os aspectos referentes à infertilidade eram negligenciados e até mesmo pouco visíveis para gestores e profissionais de saúde. Finalmente, os casos do município $\mathrm{B}$ e C são emblemáticos do quanto, em muitos contextos, é ainda a questão da esterilização cirúrgica que move os debates e as políticas no âmbito do PR e das dificuldades persistentes de colocá-lo no marco maior dos direitos reprodutivos e da integralidade na atenção à saúde. Isso se reflete no discurso de gestores e profissionais do município que com freqüência desliza entre os termos "programa de planejamento familiar" e "programa de laqueadura" quando se referem a ele.

\section{Discussão}

Os municípios estudados apresentaram graus diferenciados de implementação das ações programáticas de assistência ao PR. Contudo, alguns pontos críticos observados não apenas são comuns às três cidades, mas representam dificuldades vivenciadas por um amplo contingente de municípios brasileiros, sendo desafios para os gestores municipais, estaduais e federais. Estudos realizados na última década, alguns de alcance nacional e outros de realidades locais, evidenciaram oferta restrita e irregularidade na provisão de métodos contraceptivos reversíveis, 2,4,6,9 dificuldades de organização de referências efetivas para esterilização cirúrgica voluntária, $2-4,6,8,9$ baixa oferta de serviços e captação de adolescentes e de público masculino, 4,10 ausência de atendimento à infertilidade, $2,4,6$ pouca articulação da assistência ao planejamento reprodutivo com outras ações e programas de saúde6,7 e necessidade de capacitação de profissionais.4,6 Em relação a esse último aspecto, captação, fixação e qualificação de recursos humanos tem sido um grande desafio para o avanço da atenção básica e sua reorientação para o modelo da ESF no Brasil. 18

O componente educativo foi bem avaliado nos municípios A e C, mas seu desempenho em B foi limitado. Estudos de Nagahama ${ }^{9}$ e Heilborn et al. ${ }^{7}$ em municípios com características similares também constataram a falta de realização de atividades educativas específicas para planejamento reprodutivo, comprometendo a efetividade do programa. Em estudo de representatividade nacional, Costa et al. ${ }^{2}$ observaram que, principalmente nos municípios de menor população, não havia conjugação entre as atividades educativas em saúde e a demanda por contracepção.

Para os municípios B e C, cuja introdução das ações de assistência ao PR foi mais recente, a debilidade do componente de gerenciamento no sistema local de saúde e nas unidades é evidenciada pela ausência de registros específicos, protocolos assistenciais e mecanismos para monitoramente e avaliação, o que fragiliza a institucionalização dessas ações como um programa de saúde. Esta questão também parece ser um nó a ser desatado para a efetiva implementação do PR, principalmente quando inserido na estratégia de saúde da família, pois outro estudo de avaliação da implementação também considerou a dimensão organizacional "insatisfatória".9

$\mathrm{Na}$ análise do contexto de implementação das ações programáticas de assistência ao PR nos municípios sobressaíram alguns aspectos que condicionam a efetivação das políticas de saúde e direitos reprodutivos, e dizem respeito às desigualdades geopolíticas e ao contexto político e institucional do SUS. Os municípios do interior apresentavam dificuldades maiores do que o metropolitano em organizar a assistência ao PR, por contarem com recursos institucionais, materiais, técnicos e científicos mais escassos, além de terem menores oportunidades de participação nos debates políticos da área de saúde e interlocução com atores e instituições. Dificuldades similares que afetam municípios de menor porte, fora das áreas metropolitanas, também foram observadas em outros estudos.2,7

As dificuldades apresentadas por esses municípios de menor porte se amplificavam pela incipiência de articulação com outros municípios de suas respectivas regiões geográficas, onde as iniciativas de consórcios regionais apenas começavam. O pacto pela Saúde 2006 reconheceu que esse ainda é um desafio para o SUS em todo o território nacional e diagnosticou que "a gestão descentralizada da saúde ainda enfrenta o desafio de encontrar mecanismos que superem a fragmentação de estruturas municipais muitas vezes atomizadas, com iniqüidades na qualidade e na oferta de acesso, o que compromete a legitimidade do sistema".19 A regionalização solidária e cooperativa, proposta como diretriz para aperfeiçoar o processo de descentralização, pode ser estratégia importante para avançar na implementação da assistência ao PR, com melhor organização da oferta do conjunto de ações previstas, ampliação do acesso, garantia de integralidade da atenção e otimização de recursos, inclusive os humanos, técnicos e científicos.

Outro aspecto do contexto institucional que representava um obstáculo ao processo de implementação do PR nos municípios diz respeito às dificuldades de integração da rede assistencial. A fra- 
gilidade da articulação entre demandas oriundas dos programas da atenção básica e a oferta da assistência de média complexidade também persiste como desafio para o SUS, limitando a capacidade do sistema em atender as necessidades e resolver os problemas trazidos pelos usuários. ${ }^{20,21}$ Esse aspecto foi bastante evidenciado no município A que contava com uma rede ampla de serviços, em todos os níveis de complexidade, mas onde, muitas vezes, profissionais da atenção básica tinham dificuldades de atender e monitorar demandas como DIU, infertilidade e esterilização cirúrgica, em função da inexistência ou inconsistência dos mecanismos de referência e contra-referência. Nos municípios B e C, observamos como se entrecruzavam a questão da regionalização e da integração das ações de saúde. A assistência de média complexidade não estava suficientemente estruturada para dar conta do atendimento das demandas da atenção básica, no que diz respeito ao PR, e nem se dispunha de consórcios ou redes intermunicipais para suprir essa carência.

A implementação das ações programáticas de PR nos municípios estudados encontravam limites também nas persistentes dificuldades de superação da lógica dos programas verticais de saúde e de tradução nos processos de trabalho dos princípios da integração, coordenação e continuidade dos cuidados, que devem nortear uma política de atenção básica na perspectiva da integralidade - outro desafio premente para o SUS.21-23 Capacitar os profissionais e organizar os cuidados assistenciais de modo a captar as necessidades de PR nos programas de adolescentes, HIV/Aids, hipertensão, diabetes, hanseníase, nas consultas de pré-natal, puerpério e puericultura, nas consultas ginecológicas e clínicas é dar concretude à noção de integralidade, na perspectiva proposta por Mattos. ${ }^{24}$ As experiências dos municípios estudados deixam pendente a questão: como organizar os processos de trabalho nas redes municipais e nas unidades de saúde de modo a que a assistência ao PR não seja uma ação fragmentada e residual, mas tampouco se estruture como um programa verticalizado, em concorrência e pouco coordenado com outros programas e ações de saúde?

Por fim, a participação da gestão estadual da saúde é fator importante no processo de implementação da assistência ao PR. Alguns dos aspectos mais frágeis do programa nos municípios - provisão de contraceptivos, recursos humanos (fixação, capacitação), estruturação das referências e contra-referências - precisam ser enfrentados conjuntamente pelos gestores estaduais e municipais. Do mesmo modo, a cooperação entre eles é fundamental para avançar os processos de regionalização, integração e coorde- nação das redes de serviços e das ações de saúde, garantindo universalidade no acesso, integralidade na assistência e equidade no direito à saúde.

\section{Considerações finais}

A implementação de uma política de saúde não é conseqüência natural dos avanços em sua formalização jurídico-normativa e na elaboração de suas diretrizes maiores. Ao contrário, a implementação revela-se como um momento crucial da construção de uma política, implica outros desafios para os sistemas locais e os serviços de saúde, exigindo planejamento e estratégicas específicas, principalmente no contexto de descentralização das ações de saúde. A institucionalização das práticas de monitoramento e avaliação tem sido considerada uma dessas estratégias mais importantes. Segundo a Política Nacional de Monitoramento e Avaliação da Atenção Básica (2006), a avaliação é um componente fundamental da gestão; ela deve ser um processo contínuo e sistemático, envolver gestores, equipes de saúde e outros atores que compartilham respon-sabilidades. Seus propósitos são auxiliar a identificação de problemas e a reorientação das ações, avaliar a incorporação de novas práticas de saúde, mensurar o impacto das ações implementadas no estado de saúde da população.

O estudo apresentado pode ser considerado uma contribuição para a institucionalização da prática de monitoramento e avaliação da implementação das ações de assistência ao planejamento reprodutivo. A matriz avaliativa desenvolvida no processo do estudo permitiu uma análise mais objetiva dos programas municipais, com o estabelecimento de padrões e critérios de operacionalização que expressassem as diretrizes que regem essa política, a possibilidade de aferição do grau de implementação de cada município e de discriminação dos pontos fortes e frágeis do programa segundo componentes e dimensões e, ainda, propiciou uma base para a análise comparativa das experiências dos municípios.

O desenvolvimento de instrumentos padronizados de monitoramento e avaliação da assistência ao planejamento reprodutivo, capazes de captar realidades locais diferenciadas, gerar indicadores de implementação e apoiar avaliações de cunho regional, estadual ou nacional, pode ser uma alavanca na efetivação da Política Nacional de Direitos Sexuais e Reprodutivos. Os métodos e ferramentas desenvolvidos na presente pesquisa podem somar-se aos investimentos que tem sido feitos por autoridades, gestores, pesquisadores e 
profissionais de saúde de institucionalização das práticas de avaliação em saúde.

\section{Agradecimentos}

As autoras agradecem à Dra. Zulmira Hartz pelas contribuições dadas na etapa de desenvolvimento da

\section{Referências}

1. Brasil. Ministério da Saúde. PNDS 2006: Pesquisa Nacional Demografia e Saúde da Criança e da Mulher 2006. Brasília, DF; 2008.

2. Costa AM, Guilhem D, Silver LD. Planejamento familiar: a autonomia das mulheres sob questão. Rev Bras Saúde Matern Infant. 2006; 6: 75-84.

3. Osis MJD, Faúndes A, Makuch MY, Mello MB, Souza MH, Araújo MJO. Atenção ao planejamento familiar no Brasil hoje: reflexões sobre os resultados de uma pesquisa. Cad Saúde Pública. 2006; 22: 2481-90.

4. Berquó E, Cavenaghi S. Direitos reprodutivos de mulheres e homens face à nova legislação brasileira sobre esterilização voluntária. Cad Saúde Pública. 2003; 19 (Supl. 2) 441-53.

5. Moura ERJ, Silva RM. Qualidade da assistência em planejamento familiar na opinião de usuárias do Programa Saúde da Família. Acta Paul Enferm. 2006; 19: 150-6.

6. Moura ERJ, Silva RM, Galvão MTG. Dinâmica do atendimento em planejamento familiar no Programa Saúde da Família no Brasil. Cad Saúde Pública. 2007; 23: 961-70.

7. Heilborn ML, Portella AP, Brandão ER, Cabral CS Assistência em contracepção e planejamento reprodutivo na perspectiva de usuárias de três unidades do Sistema Único de Saúde no Estado do Rio de Janeiro, Brasil. Cad Saúde Pública. 2009; 25 (Supl. 2): S269-S78.

8. Osis MJD, Carvalho LEC, Cecatti JG, Bento SF, Pádua KS Atendimento à demanda pela esterilização cirúrgica na Região Metropolitana de Campinas, São Paulo, Brasil: percepção de gestores e profissionais dos serviços públicos de saúde. Cad Saúde Pública. 2009; 25: 625-34.

9. Nagahama ECI. Avaliação da implantação de serviços de saúde reprodutiva no município de Maringá, Paraná, Brasil Cad Saúde Pública. 2009; 25 (Supl. 2): S279-S90.

10. Vieira LM, Saes SO, Dória AAB, Goldberg TBL. Reflexões sobre a anticoncepção na adolescência no Brasil Rev Bras Saúde Matern Infant. 2006; 6: 135-40.

11. Walt G. Health policy. An introduction to process and power. New Jersey: Zed Books, London \& New Jersey; 2004.

12. Denis JL, Champagne F. A avaliação de implantação. In: Hartz ZMA, org. Avaliação em saúde: dos modelos conceituais à prática na análise da implantação de programas. Rio de Janeiro: Fiocruz; 1997. p. 49-88. metodologia e dos instrumentos de avaliação e na primeira fase de validação dos mesmos.
13. Contandriopoulos AP, Champagne F, Denis JL, Pineualt R. A avaliação na área de saúde: conceitos e métodos. In: Hartz ZMA, org. Avaliação em saúde: dos modelos conceituais à prática na análise de implantação de programas. Rio de Janeiro: Fiocruz; 1997. p. 29-47.

14. Brasil. Lei $n^{\circ} 9.263 / 1996$. Regula o $\$ 7^{\circ}$ do artigo 226 da Constituição Federal, que trata do planejamento familiar, estabelece penalidades e dá outras providências. [acesso em 14 abr 2010]. Disponível em: http://www. planalto.gov.br/ ccivil/leis/ L9263.htm

15. Brasil. Ministério da Saúde. Planejamento familiar: manual para o gestor. Brasília, DF; 2002.

16. Ministério da Saúde. Direitos sexuais e direitos reprodutivos: uma prioridade do governo. Brasília, DF; 2005.

17. Queiroz MIP. Relatos orais: do "indizível" ao "dizível”. In: Simson OM, org. Experimentos com histórias de vida (Itália-Brasil). Enciclopédia Aberta de Ciências Sociais. São Paulo: Editora Vértice; 1988. p. 14-43.

18. Conill EM. Políticas de atenção primária e reformas sanitárias: discutindo a avaliação a partir da análise do Programa Saúde da Família em Florianópolis, Santa Catarina, Brasil, 1994-2000. Cad Saúde Pública. 2002; 18 (Supl): 191-202.

19. Brasil. Ministério da Saúde. Regionalização solidária e cooperativa: orientações para sua implementação no SUS. Série Pactos pela Saúde 2006, V. 3. Brasília, DF; 2006.

20. Brasil. Ministério da Saúde. Diretrizes para a programação pactuada e integrada da assistência à saúde. Série Pactos pela Saúde 2006, V. 5. Brasília, DF; 2006.

21. Giovanella L, Mendonça MHM, Almeida PF, Escorel S, Senna MCM, Fausto MCR, Delgado MM, Andrade CLT, Cunha MS, Martins MIC, Teixeira CT. Saúde da família: limites e possibilidades para uma abordagem integral de atenção primária à saúde no Brasil. Ciênc Saúde Coletiva. 2009; 14: 783-94.

22. Brasil. Ministério da Saúde. Política Nacional de Atenção Básica. Série Pactos pela Saúde 2006, V.4. Brasília, DF; 2006.

23. Conill EM. Análisis de la problemática de la integración de la APS en el contexto actual: causas que inciden en la fragmentación de servicios y sus efectos en la cohesión social. 2007. [acesso em 14 abr 2010]. Disponível em: http://www4.ensp.fiocruz.br/biblioteca/home/exibedetalhes Biblioteca.cfm? ID $=4359 \&$ tipo $=\mathrm{B}$ 
24. Mattos RA. Os sentidos da integralidade: algumas reflexões acerca de valores que merecem ser defendidos. In: Pinheiro R, Mattos RA, org. Os sentidos da integralidade na atenção e no cuidado à saúde. Rio de Janeiro: Instituto de Medicina Social, Universidade do Estado do Rio de Janeiro/ABRASCO; 2001. p. 39-64.

Recebido em 28 de abril de 2010

Versão final apresntada em 20 de setembro de 2010

Aprovado em 20 de outubro de 2010 